\title{
Transformation of Gibbsite to Boehmite in Caustic Aqueous Solution at Hydrothermal Conditions
}

Xin Zhang ${ }^{1, *}$, Wenwen Cui ${ }^{1,2,3}$, Jian Zhi Hu${ }^{1}$, Hsiu-Wen Wang ${ }^{4}$, Micah P. Prange ${ }^{1}$, Chuan Wan ${ }^{1}$,

Nicholas R. Jaegers ${ }^{1}$, Meirong Zong ${ }^{1,5}$, Hailin Zhang ${ }^{1,2,3}$, Carolyn I. Pearce ${ }^{1}$, Ping Li ${ }^{2}$, Zheming

Wang ${ }^{1}$, Sue B. Clark ${ }^{1,6}$, and Kevin M. Rosso ${ }^{1, *}$

1 - Pacific Northwest National Laboratory, Richland, WA, USA

2 - Institute of Process Engineering, Chinese Academy of Sciences, Beijing, China

3 - University of Chinese Academy of Sciences, Beijing, China

4 - Oak Ridge National Laboratory, Oak Ridge, Tennessee 37830, United States

5 - School of Earth Sciences and Engineering, Nanjing University, Nanjing, China

6 - Department of Chemistry, Washington State University, Pullman, WA, USA 

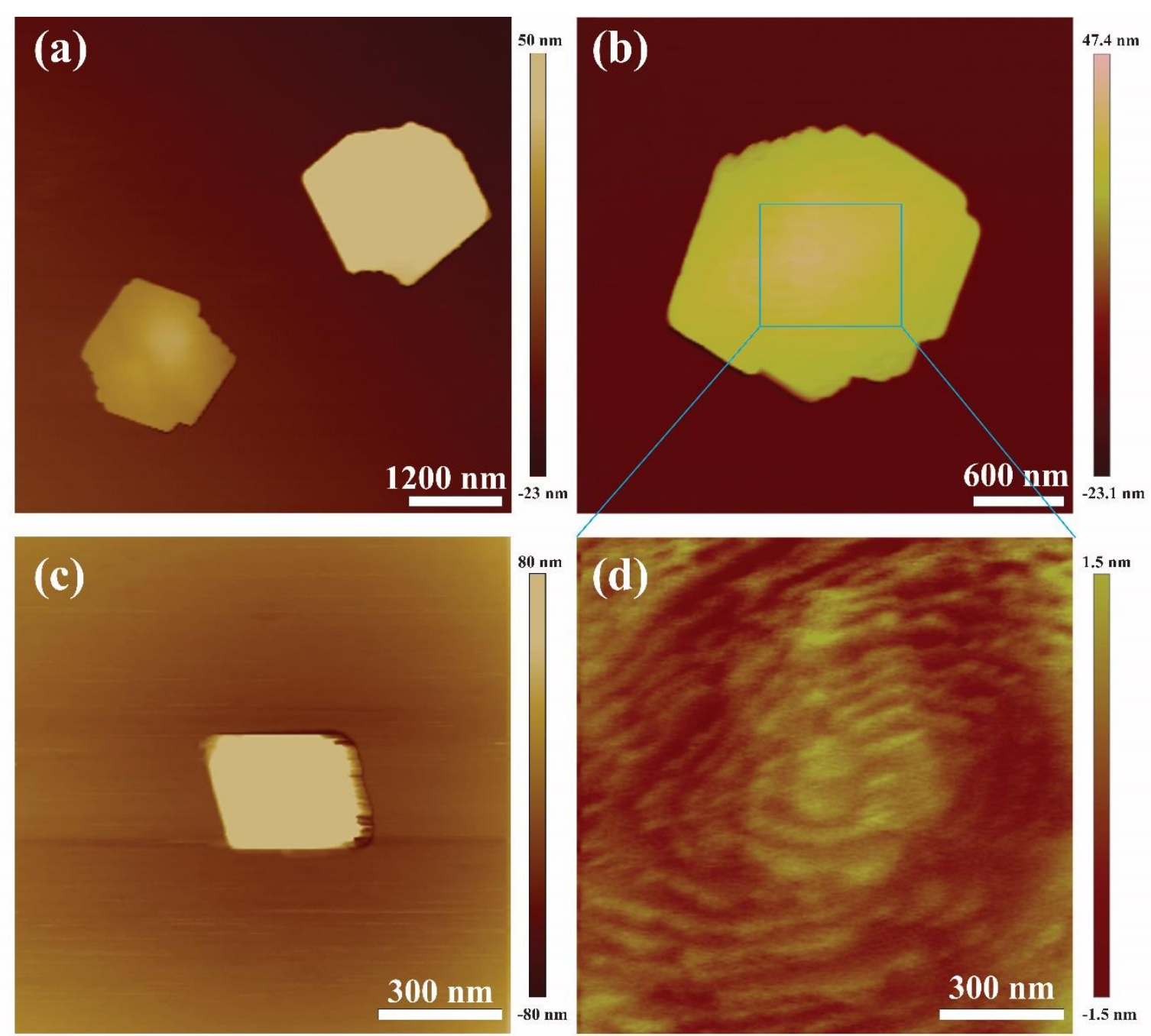

Figure S1. AFM images of boehmite produced at $100{ }^{\circ} \mathrm{C}, 10$ days (a), $120{ }^{\circ} \mathrm{C}, 2$ days (b), and 200 ${ }^{\circ} \mathrm{C}, 2$ days (c) showing (a, b) hexagonal particle shapes and (c) rhombic particle shapes. (d) AFM image of boehmite produced at $120{ }^{\circ} \mathrm{C}, 2$ days revealing spiral growth hillocks on the basal (010) surface. The concentration of precursor gibbsite and $\mathrm{NaOH}$ is $0.256 \mathrm{M}$ and $0.2 \mathrm{M}$, respectively. 


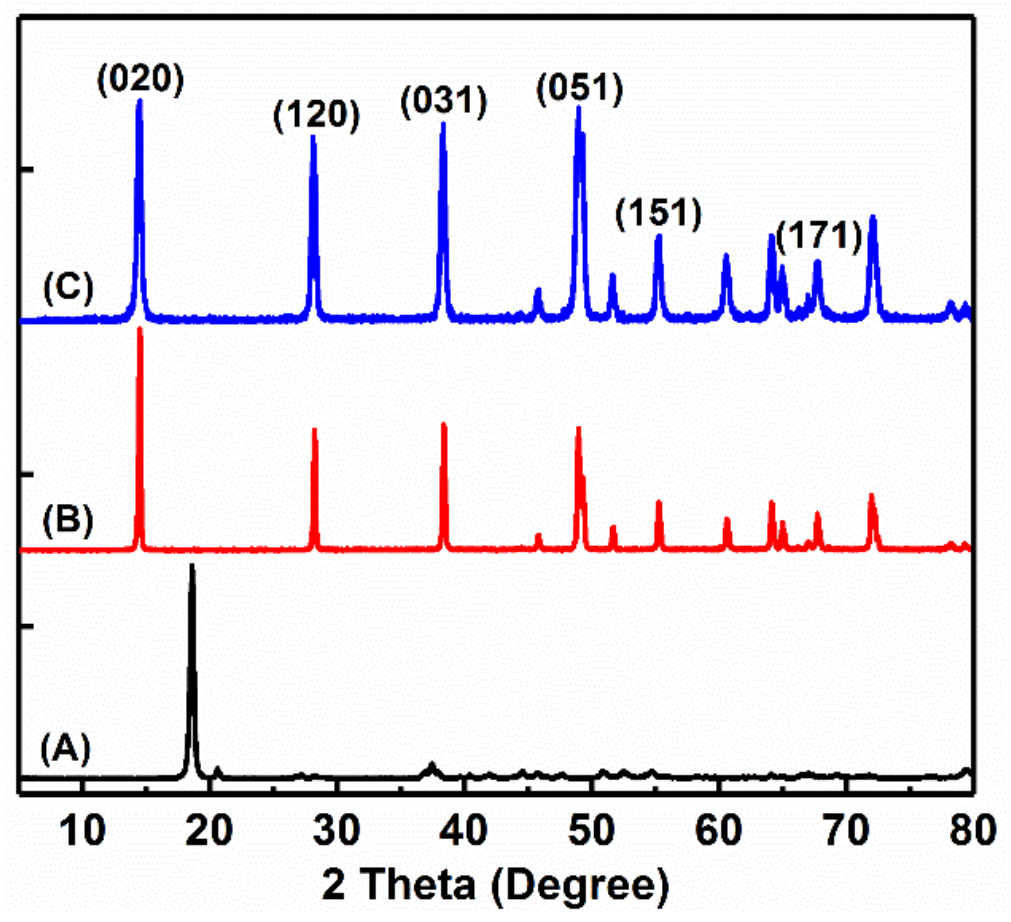

Figure S2. XRD patterns of product boehmite produced at (A) $0.01 \mathrm{M}$; (B) $0.1 \mathrm{M}$; and (C) $0.2 \mathrm{M}$ $\mathrm{NaOH}$. The temperature and reaction time for all reactions were $120^{\circ} \mathrm{C}$ and 3 days, respectively. The concentration of the precursor gibbsite was $0.256 \mathrm{M}$. 


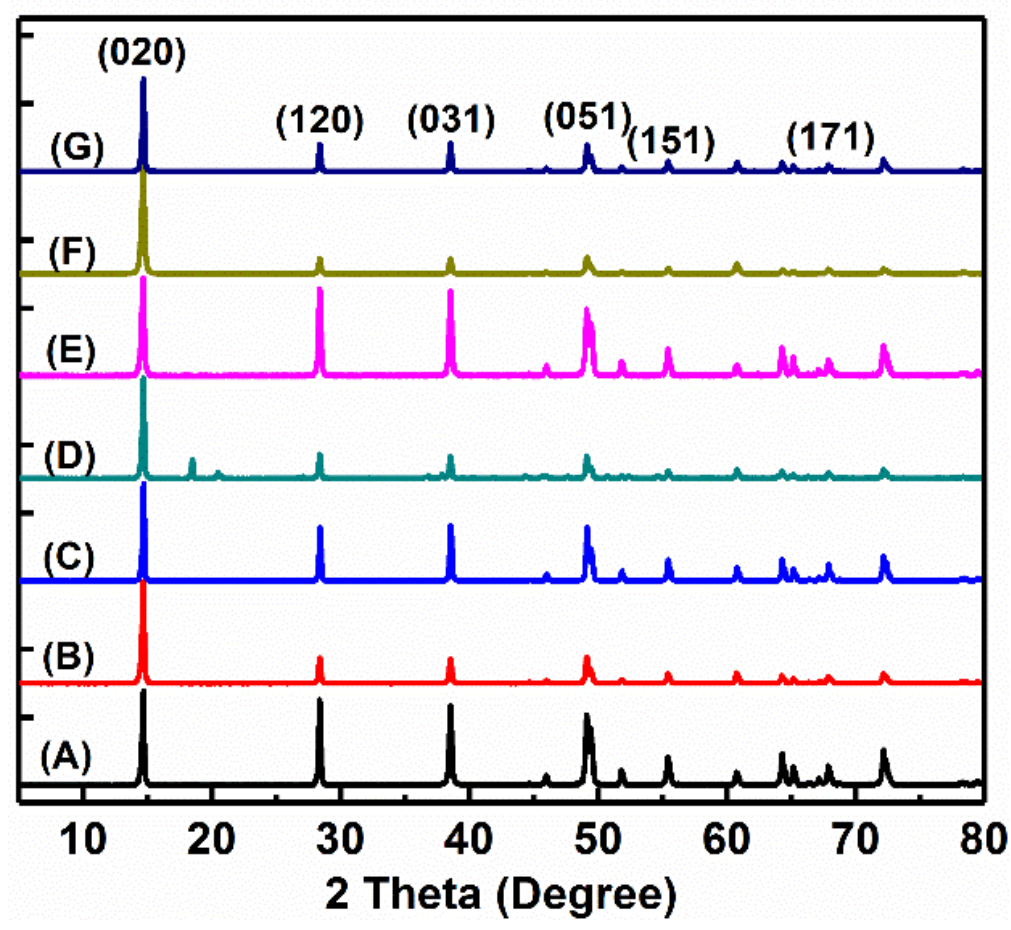

Figure S3. XRD patterns of product boehmite produced at (A) $0.064 \mathrm{M}$ Gibbsite, $0.1 \mathrm{M} \mathrm{NaOH}$, $120{ }^{\circ} \mathrm{C}$; (B) $0.25 \mathrm{M}$ Gibbsite, $0.1 \mathrm{M} \mathrm{NaOH}, 100{ }^{\circ} \mathrm{C}$; (C) $0.25 \mathrm{M}$ Gibbsite, $0.1 \mathrm{M} \mathrm{NaOH}, 120{ }^{\circ} \mathrm{C}$; (D) $0.641 \mathrm{M}$ Gibbsite, $0.2 \mathrm{M} \mathrm{NaOH}, 100{ }^{\circ} \mathrm{C}$; (E) $0.641 \mathrm{M}$ Gibbsite, $0.2 \mathrm{M} \mathrm{NaOH}, 120{ }^{\circ} \mathrm{C}$; (F) $0.641 \mathrm{M}$ Gibbsite, $0.5 \mathrm{M} \mathrm{NaOH}, 100{ }^{\circ} \mathrm{C}$; and (G) $0.641 \mathrm{M}$ Gibbsite, $0.5 \mathrm{M} \mathrm{NaOH}, 120{ }^{\circ} \mathrm{C}$. The reaction time was 10 days and 7 days for $100^{\circ} \mathrm{C}$ and $120^{\circ} \mathrm{C}$. 


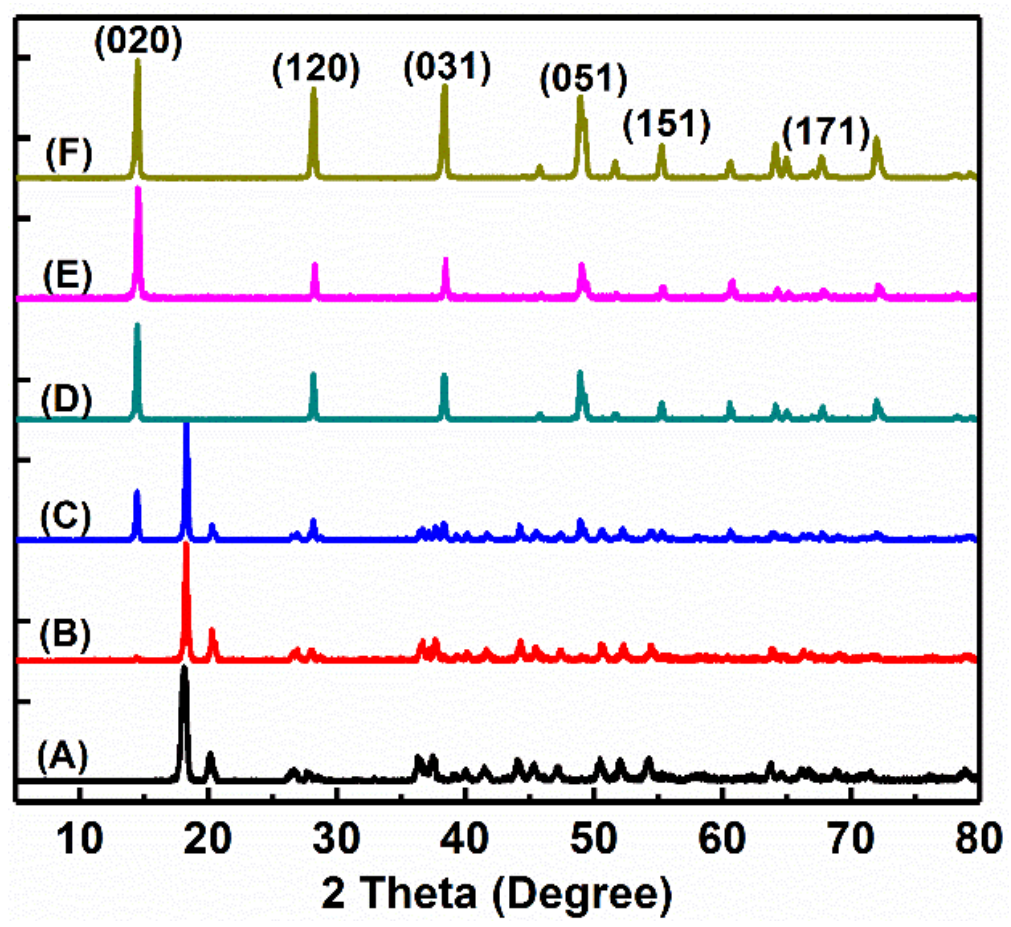

Figure S4. XRD patterns of samples synthesized at different hydrothermal treatment times: (A) 6 h; (B) $24 \mathrm{~h}$; (C) $30 \mathrm{~h}$; (D) $36 \mathrm{~h}$; (E) $42 \mathrm{~h}$; and (F) $48 \mathrm{~h}$. The concentration of gibbsite and $\mathrm{NaOH}$ was $0.256 \mathrm{M}$ and $0.2 \mathrm{M}$, respectively. The temperature was $120{ }^{\circ} \mathrm{C}$. 


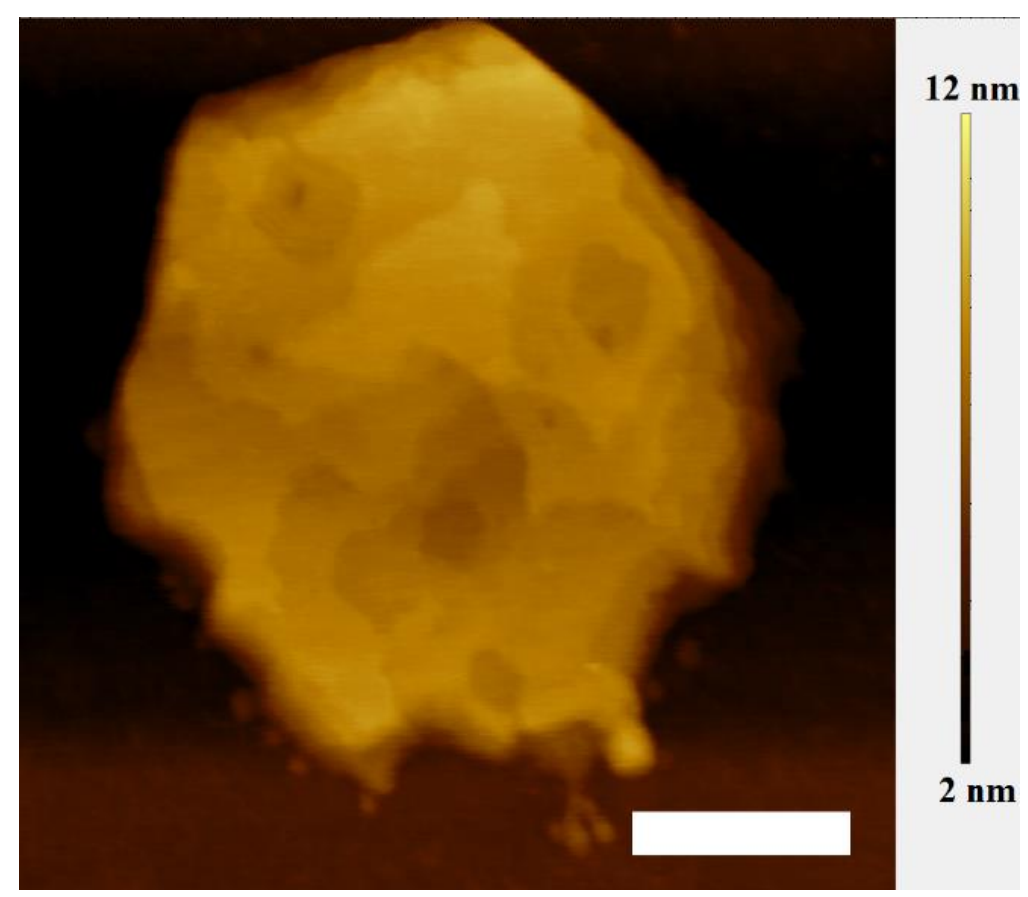

Figure S5. AFM image of sample collected at $6 \mathrm{~h}$ hydrothermal treatment time. The concentration of gibbsite and $\mathrm{NaOH}$ was $0.256 \mathrm{M}$ and $0.2 \mathrm{M}$, respectively. The temperature was $120{ }^{\circ} \mathrm{C}$. The insert scale bar is $100 \mathrm{~nm}$. 


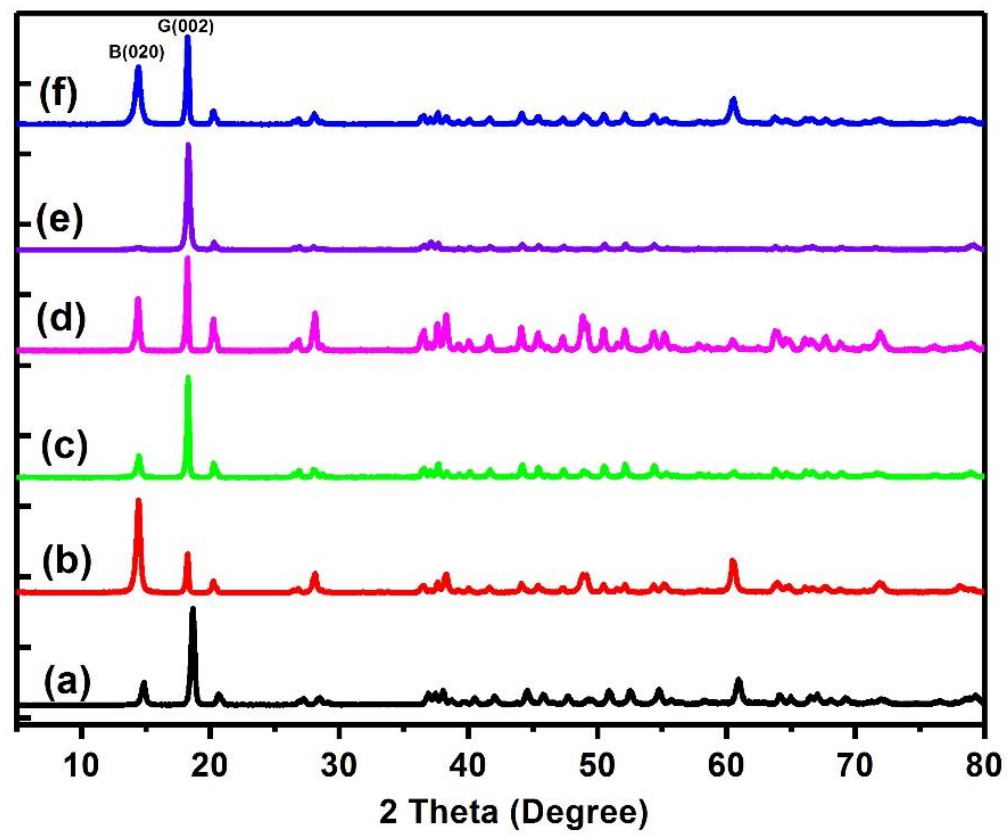

Figure S6. XRD patterns of samples synthesized with different additives: (A) $6 \mathrm{~h}$ reaction solution with $0.128 \mathrm{M} \mathrm{NaAlO}_{2}$ solution; (B) $24 \mathrm{~h}$ reaction solution with $0.128 \mathrm{M} \mathrm{NaAlO}_{2}$ solution; (C) $6 \mathrm{~h}$ reaction solution with $0.128 \mathrm{M} \mathrm{NaAc}$ solution; (D) $24 \mathrm{~h}$ reaction solution with $0.128 \mathrm{M} \mathrm{NaAc}$ solution; (E) $6 \mathrm{~h}$ reaction solution with $0.128 \mathrm{M}$ sodium oleate solution; and $(\mathrm{F}) 24 \mathrm{~h}$ reaction solution with $0.128 \mathrm{M}$ sodium oleate solution. The $6 \mathrm{~h}$ and $24 \mathrm{~h}$ reaction solution was prepared by using the conditions: concentration of gibbsite and $\mathrm{NaOH}$ was $0.256 \mathrm{M}$ and $0.2 \mathrm{M}$, respectively; temperature was $120^{\circ} \mathrm{C}$, reaction time was $6 \mathrm{~h}$ and $24 \mathrm{~h}$, respectively. 

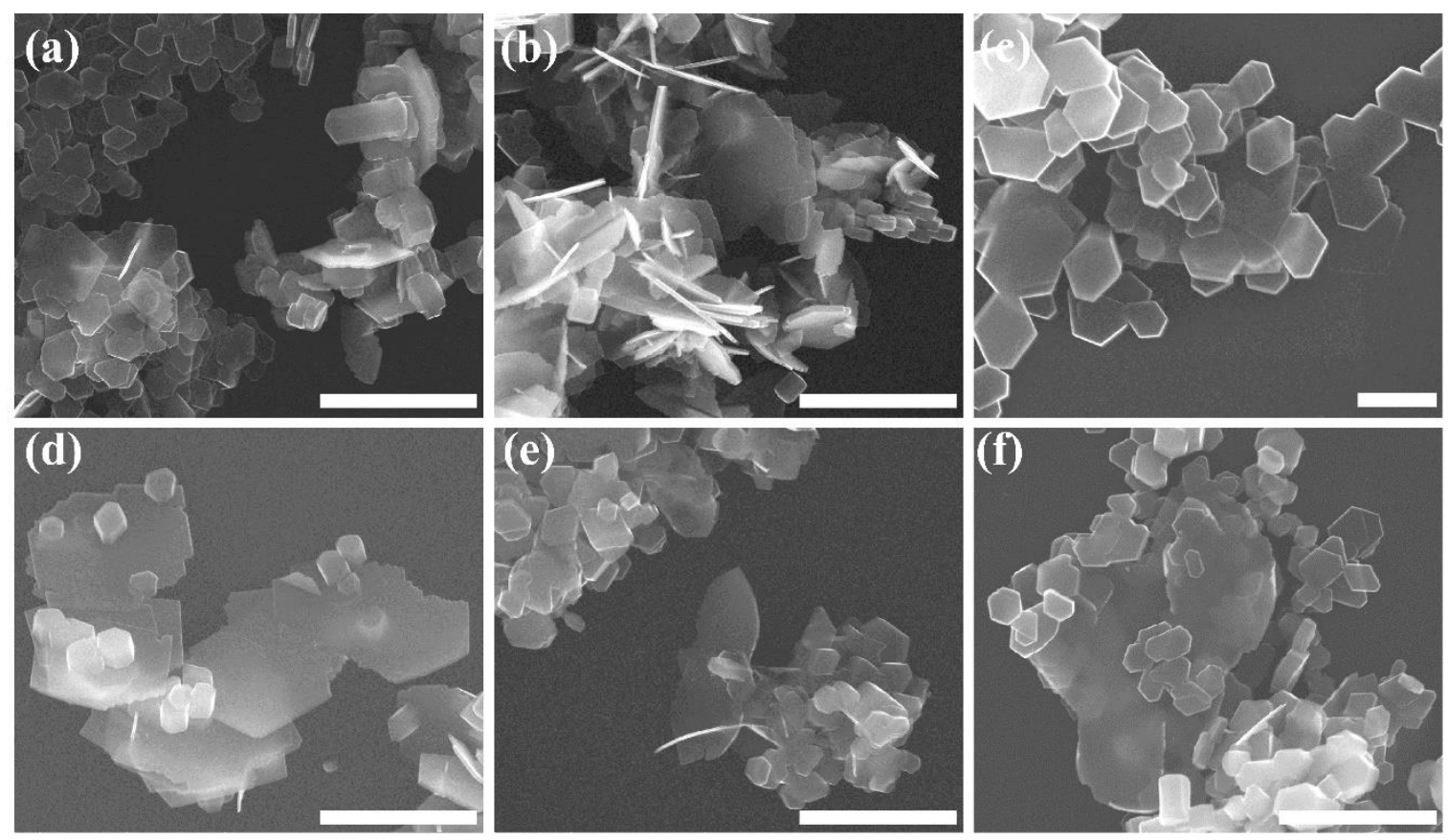

Figure S7. SEM images of samples synthesized with different additives: (A) $6 \mathrm{~h}$ reaction solution with $0.128 \mathrm{M} \mathrm{NaAlO}_{2}$ solution; (B) $24 \mathrm{~h}$ reaction solution with $0.128 \mathrm{M} \mathrm{NaAlO}_{2}$ solution; (C) $6 \mathrm{~h}$ reaction solution with $0.128 \mathrm{M} \mathrm{NaAc}$ solution; (D) $24 \mathrm{~h}$ reaction solution with $0.128 \mathrm{M} \mathrm{NaAc}$ solution; (E) $6 \mathrm{~h}$ reaction solution with $0.128 \mathrm{M}$ sodium oleate solution; and $(\mathrm{F}) 24 \mathrm{~h}$ reaction solution with $0.128 \mathrm{M}$ sodium oleate solution. The $6 \mathrm{~h}$ and $24 \mathrm{~h}$ reaction solution was prepared by using the conditions: concentration of gibbsite and $\mathrm{NaOH}$ was $0.256 \mathrm{M}$ and $0.2 \mathrm{M}$, respectively; temperature was $120^{\circ} \mathrm{C}$, reaction time was $6 \mathrm{~h}$ and $24 \mathrm{~h}$, respectively. The scale bar is $2 \mu \mathrm{m}$. 
Table S1. Fitting Results of Figure 10.

\begin{tabular}{|c|c|c|c|c|c|c|}
\hline Sample name & Site name & $\begin{array}{c}\text { Chemical shift } \\
(\text { ppm })\end{array}$ & $\begin{array}{c}\mathrm{CQ} \\
(\mathrm{MHz})\end{array}$ & $\eta$ & $\begin{array}{c}\mathbf{L b} \\
(\mathbf{p p m})\end{array}$ & $\begin{array}{c}\text { Area ratio } \\
(\%)\end{array}$ \\
\hline \multirow{4}{*}{$\begin{array}{l}\text { Precursor: } \\
\text { gibbsite }\end{array}$} & Octa-site & 11.3 & 2.6 & 1.0 & 2.7 & 99.51 \\
\hline & Penta-site & 41.7 & 6.7 & 0.6 & 6.4 & 0.40 \\
\hline & \multirow{2}{*}{ Tetra-site } & - & - & - & - & - \\
\hline & & 74.8 & 4.8 & 0.5 & 8.0 & 0.09 \\
\hline \multirow{4}{*}{$\begin{array}{l}6 \mathrm{~h} \text { sample: } \\
100 \% \text { gibbsite }\end{array}$} & Octa-site & 11.2 & 2.6 & 1.0 & 2.7 & 98.42 \\
\hline & Penta-site & 42.8 & 7.0 & 0.3 & 9.7 & 0.98 \\
\hline & \multirow{2}{*}{ Tetra-site } & 61.9 & 4.4 & 1.0 & 10.5 & 0.11 \\
\hline & & 73.3 & 4.9 & 0.8 & 12.0 & 0.50 \\
\hline \multirow{4}{*}{$\begin{array}{l}24 \text { h sample: } \\
97 \% \text { gibbsite and } \\
3 \% \text { boehmite }\end{array}$} & Octa-site & 11.2 & 2.6 & 1.0 & 2.7 & 98.66 \\
\hline & Penta-site & 42.7 & 6.8 & 0.6 & 8.0 & 0.94 \\
\hline & \multirow{2}{*}{ Tetra-site } & 62.0 & 4.4 & 1.0 & 10.3 & 0.06 \\
\hline & & 74.1 & 4.8 & 0.5 & 10.3 & 0.33 \\
\hline \multirow{4}{*}{$\begin{array}{l}30 \text { h sample, } \\
70 \% \text { gibbsite and } \\
30 \% \text { boehmite }\end{array}$} & Octa-site & 11.1 & 2.6 & 1.0 & 2.7 & 99.3 \\
\hline & Penta-site & 41.7 & 6.7 & 0.6 & 6.4 & 0.36 \\
\hline & \multirow{2}{*}{ Tetra-site } & 62.0 & 4.3 & 1.0 & 9.3 & 0.05 \\
\hline & & 73.2 & 4.8 & 0.5 & 10.3 & 0.25 \\
\hline \multirow{4}{*}{$\begin{array}{l}36 \text { h sample: } \\
100 \% \text { boehmite }\end{array}$} & Octa-site & 11.1 & 2.6 & 1.0 & 2.7 & 99.61 \\
\hline & Penta-site & 42.2 & 6.7 & 0.5 & 6.4 & 0.19 \\
\hline & \multirow{2}{*}{ Tetra-site } & 62.0 & 4.4 & 1.0 & 10.7 & 0.03 \\
\hline & & 73.0 & 4.9 & 0.5 & 11.1 & 0.17 \\
\hline \multirow{4}{*}{$\begin{array}{l}42 \text { h sample: } \\
100 \% \text { boehmite }\end{array}$} & Octa-site & 10.7 & 2.6 & 1.0 & 3.9 & 99.61 \\
\hline & Penta-site & 41.5 & 6.7 & 0.5 & 5.4 & 0.18 \\
\hline & \multirow{2}{*}{ Tetra-site } & 62.0 & 4.3 & 1.0 & 9.5 & 0.03 \\
\hline & & 72.9 & 4.8 & 0.5 & 10.0 & 0.17 \\
\hline
\end{tabular}


Table S2. Overview of boehmite synthesis conditions and resulting products with secondary $\mathrm{Al}(\mathrm{III})$ precursor and/or organic additives.

\begin{tabular}{|c|c|c|c|c|c|c|c|c|c|}
\hline \multirow{2}{*}{ Entry } & \multirow{2}{*}{$\begin{array}{c}{\left[\mathrm{Al}^{3+}\right]} \\
\text { (M, based } \\
\text { on gibbsite) }\end{array}$} & \multirow{2}{*}{$\begin{array}{c}{[\mathrm{NaOH}]} \\
(\mathrm{M})\end{array}$} & \multirow{2}{*}{$\begin{array}{l}\text { Temp. } \\
\left({ }^{\circ} \mathrm{C}\right)\end{array}$} & \multirow{2}{*}{$\begin{array}{l}\text { Time } \\
\text { (h) }\end{array}$} & \multirow{2}{*}{ Additive } & \multirow{2}{*}{$\begin{array}{l}\text { Temp. } \\
\left({ }^{\circ} \mathrm{C}\right)\end{array}$} & \multirow{2}{*}{$\begin{array}{l}\text { Time } \\
\text { (h) }\end{array}$} & \multicolumn{2}{|c|}{ Product } \\
\hline & & & & & & & & Boehmite & Gibbsite \\
\hline 1 & 0.256 & 0.2 & 120 & 6 & $\begin{array}{c}\mathrm{NaAlO}_{2}(\mathrm{aq}) \\
0.128 \mathrm{M}\end{array}$ & 120 & 48 & $19.4 \%$ & $80.6 \%$ \\
\hline 2 & 0.256 & 0.2 & 120 & 24 & $\begin{array}{c}\mathrm{NaAlO}_{2}(\mathrm{aq}) \\
0.128 \mathrm{M}\end{array}$ & 120 & 48 & $81.3 \%$ & $18.7 \%$ \\
\hline 3 & 0.256 & 0.2 & 120 & 6 & $\begin{array}{c}\mathrm{NaAc}(\mathrm{aq}) \\
0.128 \mathrm{M}\end{array}$ & 120 & 48 & $24.5 \%$ & $75.5 \%$ \\
\hline 4 & 0.256 & 0.01 & 120 & 24 & $\begin{array}{c}\mathrm{NaAc}(\mathrm{aq}) \\
0.128 \mathrm{M}\end{array}$ & 120 & 48 & $44.2 \%$ & $55.8 \%$ \\
\hline 5 & 0.256 & 0.1 & 120 & 6 & $\begin{array}{c}\text { Sodium } \\
\text { oleate }(\mathrm{aq}) \\
0.128 \mathrm{M}\end{array}$ & 120 & 48 & $1.3 \%$ & $98.7 \%$ \\
\hline 6 & 0.256 & 0.2 & 120 & 24 & $\begin{array}{c}\text { Sodium } \\
\text { oleate (aq) } \\
0.128 \mathrm{M}\end{array}$ & 120 & 48 & $56.3 \%$ & $43.7 \%$ \\
\hline
\end{tabular}

